\title{
COMPOSIÇÃO MINERAL DE SUCOS CONCENTRADOS DE FRUTAS BRASILEIRAS ${ }^{1}$
}

\author{
Lucia M. ValenteSOARES ${ }^{2, *}$, Katia SHISHIDO², Adriana M. Monteiro MORAES²,
}

\author{
Valéria Avila MOREIRA ${ }^{2}$
}

\section{RESUMO}

Informações sobre a composição de alimentos nacionais são escassas. Sucos concentrados de frutas são amplamente utilizados por famílias brasileiras. No presente trabalho foram determinados oito elementos minerais (K, $\mathrm{Na}, \mathrm{Ca}, \mathrm{Mg}, \mathrm{Fe}, \mathrm{Zn}, \mathrm{Cu}, \mathrm{Mn})$ com importância nutricional em sucos concentrados comerciais de frutas nacionais, abacaxi ( 3 marcas), acerola (2 marcas), caju (5 marcas), goiaba (3 marcas), manga (2 marcas), maracujá (5 marcas) e pitanga (1 marca) por espectrometria de absorção atômica com atomização em chama. A contribuição nutricional dos sucos para a dieta de crianças após a diluição recomendada pelo fabricante e considerando a ingestão diária de um copo de $300 \mathrm{~mL}$ é elevada quanto a potássio para todos os sucos examinados (170 - 930\% da ingestão diária recomendada, RDA). Os sucos de abacaxi e acerola podem fornecer 6 e $12 \%$ da RDA de ferro, respectivamente. O manganês aparece em abacaxi, manga, goiaba e acerola com 38, 14, 8 e 7\% do RDA, respectivamente. Magnésio varia entre um máximo de 9\% do RDA em abacaxi e $2 \%$ em maracujá e caju. Zinco e cobre variaram entre menos de $1-2 \%$ do RDA em sucos de caju e pitanga, a $2-6 \%$ nos outros sucos. Para adultos as contribuições são proporcionalmente menores, porém em nada desprezíveis.

Palavras-chave: frutas nacionais; sucos de fruta; composição mineral; minerais essenciais; potássio; ferro.

\section{SUMMARY}

MINERAL COMPOSITION OF BRAZILIAN CONCENTRATE FRUIT JUICES. Information on the composition of Brazilian foods is scant. Concentrate fruit juices commercialized in glass bottles are widely used by Brazilian families. The present work eight nutritionally important elements (K, Na, Ca, Mg, Fe, $\mathrm{Zn}, \mathrm{Cu}, \mathrm{Mn}$ ) in commercial concentrated fruit juices, pineapple ( 3 brands), West Indian cherry ( 2 brands), cashew ( 5 brands), guava ( 3 brands), mango ( 2 brands), passion fruit ( 5 brands), and Surinam cherry ( 1 brand) were determined by flame atomic absorption spectrometry. The juices contribution to children's diet, considering an intake of $300 \mathrm{~mL}$ per day and using the dilution suggested by the maker, is high in potassium for all juices analyzed (170-930\% of the recommended daily allowance, RDA). The pineapple and West Indian cherry juices offer 6 and $12 \%$ of the iron RDA, respectively. The manganese present in pineapple, mango, guava, and West Indian cherry juices contribute with 38, 14, 8 e $7 \%$ of the RDA, respectively. Magnesium varies between $9 \%$ of the RDA in pineapple juice and $2 \%$ in the passion fruit and cashew ones. Zinc and copper varied between less then $1-2 \%$ of the RDA in cashew and Surinam cherry juices and 2 to $6 \%$ in the other juices. For adults the contribution to the diet is proportionately less but by no means to be disregarded.

Keywords: brazilian fruits; fruit juices; mineral composition; essential minerals; potassium; iron.

\section{1 - INTRODUÇÃO}

Os elementos minerais reconhecidos como essenciais são comumente divididos entre macroelementos (cálcio, fósforo, potássio, sódio, cloro, magnésio, enxofre) e microelementos (ferro, cobre, cobalto, manganês, zinco, iodo, flúor, molibdênio, selênio, cromo, silício), de acordo com as quantidades maiores ou menores em que são encontrados no organismo humano. A importância de sua inclusão na dieta tem sido amplamente discutida em textos sobre nutrição [10].

Sucos concentrados de frutas nacionais, embalados em garrafas de vidro ou de plástico, são populares com famílias brasileiras e seu armazenamento é conveniente por não requerer refrigeração antes da sua utilização. Este tipo de embalagem possibilita seu transporte e comercialização em todo o território nacional. Os sucos de frutas, bebidas lácteas e refrigerantes são consumidos por crianças e adultos. Informação nutricional torna-se necessária para que as famílias sejam

\footnotetext{
1. Recebido para publicação em 25/05/2002. Aceito para publicação em 12/05/2004 (0000864).

2. Faculdade de Engenharia de Alimentos, Universidade Estadual de Campinas - UNICAMP. Caixa Postal 6121 - CEP 13084-970-Campinas, SP. E-mail:valente@fea.unicamp.br

* A quem a correspondência deve ser enviada.
}

orientadas e possam melhor balancear suas dietas com relação à ingestão de bebidas.

Informações sobre a composição de alimentos nacionais são em geral escassas. Sucos de uva produzidos no Estado do Rio Grande do Sul foram extensivamente analisados (53 amostras) e os teores dos elementos minerais mais importantes (K, Na, Ca, Mg, $\mathrm{Fe}, \mathrm{Zn}, \mathrm{Cu}, \mathrm{Mn}, \mathrm{Li}$ e P) determinados [9]. Os elementos minerais (K, Na, Ca, Mg, Fe, Zn, Cu, Mn e P) foram também determinados em sucos de frutas diversos (abacaxi, acerola, caju, goiaba, manga, maracujá e uva) provenientes de um único fabricante [6]. No exterior poucos estudos da composição de sucos de frutas tropicais comerciais têm sido efetuados [1,5].

O presente trabalho visou determinar oito elementos minerais essenciais $(\mathrm{K}, \mathrm{Na}, \mathrm{Ca}, \mathrm{Mg}, \mathrm{Fe}, \mathrm{Zn}, \mathrm{Cu}$, $\mathrm{Mn})$ em sucos concentrados comerciais de frutas nacionais, de várias marcas disponíveis no mercado, de maneira a fornecer informação sobre sua composição.

\section{2 - MATERIAIS E MÉTODOS}

\section{1 - Amostras}

Sucos concentrados de frutas, de marcas diversas e embalados em garrafas de vidro, foram adquiridos em lojas e supermercados da região de Campinas, S.P. Os 
sucos foram conservados à temperatura ambiente até análise. Foram analisados sucos de abacaxi (3 marcas), cajú (5 marcas), goiaba (3 marcas), manga (2 marcas), maracujá (5 marcas) e pitanga (1 marca). Os sucos comerciais de frutas nacionais eram provenientes de 7 fabricantes e todas as marcas eram de circulação nacional.

\section{2 - Determinação da densidade dos sucos}

As amostras de sucos foram analisadas por picnometria para determinação de sua densidade.

\section{3 - Preparo de padrões}

Foram utilizadas ampolas de padrões para absorção atômica (Carlo Erba ou Merck). Os padrões foram diluídos com água deionizada (MilliQ plus, Millipore) e soluções de ácido nítrico e de lantânio de maneira que na solução final contivessem $1 \%$ e $0,5 \%$, respectivamente.

\section{4 - Limpeza de vidraria, recipientes plásticos e demais utensilios}

Após lavagem com água e detergente, foram bem enxaguados e deixados de molho durante a noite em solução 10\% de ácido nítrico, preparada com água destilada. Ao retirar, foram escorridos e lavados pelo menos 3 vezes em água deionizada. Foram então deixados secar em posição invertida e ao abrigo de poeira.

\section{5 - Abertura das amostras}

Foram pesados $10 \mathrm{~g}$ do suco em tubo de $25 \mathrm{~cm}$ x $250 \mathrm{~mm}$ de borda reforçada e $10 \mathrm{~mL}$ de ácido nítrico (Merck, p.a.) foram adicionados. As amostras foram mineralizadas em digestor infravermelho (Modelo 4002, Marconi). Após digeridas e secas, foram re-suspendidas em solução 5\% lantânio (óxido de lantânio, Reacton, Alfa Aesar) e solução 10\% de ácido nítrico (Merck, p.a.). Foram, a seguir, transferidas para balão volumétrico de maneira que a concentração final contivesse $1 \%$ de ácido nítrico e 0,5\% de lantânio. Água deionizada foi usada para o preparo das soluções e para as diluições. Brancos foram preparados com o mesmo procedimento.

As amostras de referência certificadas foram mineralizadas como descrito acima, porém, a adição de ácido nítrico concentrado foi repetida o necessário para incineração total das amostras. Brancos foram preparados com a mesma quantidade de ácido nítrico concentrado.

\section{6 - Determinação dos teores dos elementos mi- nerais}

Foi utilizado um espectrofotômetro de absorção atômica (modelo 5100 PC, Perkin Elmer) com módulo de atomização em chama, pérola de impacto e correção de fundo com lâmpada de deutério. Misturas acetileno/ar foram empregadas nas proporções recomendadas pelo fabricante do instrumento para os diferentes elementos. As linhas espectrais escolhidas foram as princi- pais, exceto para potássio e magnésio $(769,9 \mathrm{~nm}$ para $\mathrm{K}$ e 202,6nm para $\mathrm{Mg}$ ) por questões de conveniência com relação à concentração destes elementos nos sucos (Tabela 1). A altura do queimador foi otimizada para maior sensibilidade.

TABELA 1. Comprimentos de onda empregados, limites de detecção e repetibilidade das determinações dos elementos minerais nas amostras de suco concentrado

\begin{tabular}{llll}
\hline Elemento & $\lambda(\mathrm{nm})$ & L.D. $(\mathrm{mg} / \mathrm{L})$ & CV médio $(\%)$ \\
\hline $\mathrm{K}$ & 769,9 & 0,03 & 3,7 \\
$\mathrm{Na}$ & 589,0 & 0,012 & 0,9 \\
$\mathrm{Ca}$ & 422,7 & 0,04 & 3,6 \\
$\mathrm{Mg}$ & 285,2 & 0,14 & 2,0 \\
$\mathrm{Fe}$ & 248,3 & 0,02 & 5,7 \\
$\mathrm{Zn}$ & 213,9 & 0,012 & 6,9 \\
$\mathrm{Cu}$ & 324,8 & 0,012 & 2,0 \\
$\mathrm{Mn}$ & 202,6 & 0,02 & 0,7
\end{tabular}

L.D. - limite de detecção (3 vezes o desvio padrão de 5 leituras do branco). CV médio (\%) - coeficiente de variação médio das determinações em duplicata das amostras.

\section{7 - Controle de qualidade analítico}

As seguintes amostras de referência certificadas foram adquiridas do National Institute of Standards and Tecnology, Gaithersburg, EUA: Wheat Flour SRM 1567a, Apple Leaves SRM 1515, Peach Leaves SRM 1547.

Uma amostra de referência doméstica foi preparada misturando 5 litros de suco de uva em um recipiente plástico. Após a mistura o suco foi transferido para frascos de polietileno de cerca de $200 \mathrm{~mL}$. Os frascos com o suco foram mantidos até utilização a $-18^{\circ} \mathrm{C}$. A amostra de referência doméstica foi analisada para os elementos de interesse com 15 repetições realizadas em 3 dias diferentes. Foram calculados, para cada elemento, a média e o desvio padrão dos resultados.

Validação das condições de trabalho: foram determinados os elementos minerais de interesse nas amostras de referência certificadas e os resultados comparados com os valores certificados para verificação da exatidão do procedimento. O critério de aceitação dos resultados foi estarem dentro do desvio padrão expresso na certificação.

Validação dos resultados de cada série de sucos analisada: em cada série de amostras analisadas, incluiu-se a amostra de referência preparada no laboratório e um branco. Os resultados da amostra de referência doméstica foram verificados contra os resultados previamente estabelecidos para a mesma para cada elemento. Os resultados que concordaram dentro de um desvio padrão com os previamente estabelecidos indicaram a aceitabilidade dos resultados da batelada de amostras de sucos analisadas. 


\section{3 - RESULTADOS E DISCUSSÃO}

As amostras de referência certificadas foram usadas para verificar a exatidão do procedimento. Os resultados obtidos estavam dentro do desvio certificado para todos os elementos determinados. Todas as séries de amostras analisadas foram acompanhadas de uma amostra de referência doméstica (suco de uva) e um branco. Foram consideradas aceitáveis as séries de resultados cuja amostra de referência doméstica apresentou teores para todos os elementos dentro de um desvio padrão dos resultados já anteriormente estabelecidos no laboratório para a amostra de referência. Os limites de detecção nas condições de trabalho empregadas estão na Tabela 1 . O coeficiente de variação para cada conjunto de duplicatas analisadas foi calculado para expressar a repetibilidade (precisão) dos procedimentos (Tabela 1) e mostraram-se adequados.

TABELA 2. Concentrações de elementos minerais em sucos concentrados de abacaxi

\begin{tabular}{|c|c|c|c|c|c|c|c|c|c|}
\hline \multirow[t]{2}{*}{ Marca } & \multicolumn{7}{|c|}{ Teores de elementos minerais $(\mathrm{mg} / 100 \mathrm{~g})$} & \multicolumn{2}{|r|}{$\begin{array}{r}\text { Densidade } \\
\left(\mathrm{g} / \mathrm{cm}^{3}\right)\end{array}$} \\
\hline & $\mathrm{K}$ & $\mathrm{Na}$ & $\mathrm{Ca}$ & $\mathrm{Mg}$ & $\mathrm{Fe}$ & $\mathrm{Zn}$ & $\mathrm{Cu}$ & $\mathrm{Mn}$ & \\
\hline A & 606 & 12,3 & 77,7 & 50,3 & 2,57 & 0,48 & 0,17 & 1,23 & 1,048 \\
\hline B & 149 & 28,5 & 5,4 & 7,0 & 0,15 & 0,08 & 0,04 & 0,07 & 1,049 \\
\hline C & 123 & 28,2 & 18,0 & 13,1 & 0,34 & 0,07 & 0,04 & 0,74 & 1,040 \\
\hline G & 107 & 13,8 & 18,8 & 12,7 & 0,34 & 0,40 & 0,05 & 0,37 & 1,032 \\
\hline Teores médios & 246 & 18,2 & 29,9 & 20,8 & 0,85 & 0,26 & 0,08 & 0,60 & 1,042 \\
\hline
\end{tabular}

TABELA 3. Concentrações de elementos minerais em sucos concentrados de acerola

\begin{tabular}{lrrrrrrrrrr}
\hline Marca & \multicolumn{1}{c}{ Teores de elementos minerais $(\mathrm{mg} / 100 \mathrm{~g})$} & & $\begin{array}{r}\text { Densidade } \\
\left(\mathrm{g} / \mathrm{cm}^{3}\right)\end{array}$ \\
\hline & $\mathrm{K}$ & $\mathrm{Na}$ & $\mathrm{Ca}$ & $\mathrm{Mg}$ & $\mathrm{Fe}$ & $\mathrm{Zn}$ & $\mathrm{Cu}$ & $\mathrm{Mn}$ & \\
\hline $\mathrm{B}$ & 117 & 20,6 & 10,1 & 9,1 & 2,94 & 0,09 & 0,04 & 0,16 & 1,021 \\
$\mathrm{C}$ & 91 & 21,0 & 4,2 & 6,8 & 0,29 & 0,06 & 0,02 & 0,03 & 1,012 \\
\hline Teores médios & 104 & 20,8 & 7,2 & 8,0 & 1,60 & 0,08 & 0,03 & 0,10 & 1,016 \\
\hline Os resultados representam médias de duplicatas. & & & & & & &
\end{tabular}

TABELA 4. Concentrações de elementos minerais em sucos concentrados de caju

\begin{tabular}{|c|c|c|c|c|c|c|c|c|c|}
\hline \multirow[t]{2}{*}{ Marca } & \multicolumn{7}{|c|}{ Teores de elementos minerais $(\mathrm{mg} / 100 \mathrm{~g})$} & \multicolumn{2}{|r|}{$\begin{array}{r}\text { Densidade } \\
\left(\mathrm{g} / \mathrm{cm}^{3}\right)\end{array}$} \\
\hline & $\mathrm{K}$ & $\mathrm{Na}$ & $\mathrm{Ca}$ & $\mathrm{Mg}$ & $\mathrm{Fe}$ & $\mathrm{Zn}$ & $\mathrm{Cu}$ & $\mathrm{Mn}$ & \\
\hline A & 115 & 39,4 & 0,7 & 8,9 & 0,18 & 0,09 & 0,05 & 0,07 & 1,053 \\
\hline B & 132 & 41,2 & 0,8 & 11,1 & 0,44 & 0,12 & 0,05 & 0,10 & 1,050 \\
\hline C & 112 & 37,6 & 0,5 & 8,7 & 0,18 & 0,09 & 0,04 & 0,06 & 1,045 \\
\hline E & 120 & 31,2 & 0,6 & 8,9 & 0,16 & 0,08 & 0,05 & 0,06 & 1,051 \\
\hline $\mathrm{F}$ & 98 & 32,6 & 0,7 & 7,7 & 0,46 & 0,12 & 0,04 & 0,07 & 1,035 \\
\hline Teores médios & 115 & 36,4 & 0,7 & 9,1 & 0,29 & 0,10 & 0,05 & 0,07 & 1,047 \\
\hline
\end{tabular}

Os resultados representam médias de duplicatas.
TABELA 5. Concentrações de elementos minerais em sucos concentrados de goiaba

\begin{tabular}{|c|c|c|c|c|c|c|c|c|c|}
\hline \multirow[t]{2}{*}{ Marca } & \multicolumn{7}{|c|}{ Teores de elementos minerais (mg/100g) } & \multicolumn{2}{|r|}{$\begin{array}{r}\text { Densidade } \\
\left(\mathrm{g} / \mathrm{cm}^{3}\right)\end{array}$} \\
\hline & $\mathrm{K}$ & $\mathrm{Na}$ & $\mathrm{Ca}$ & $\mathrm{Mg}$ & $\mathrm{Fe}$ & $\mathrm{Zn}$ & $\mathrm{Cu}$ & $\mathrm{Mn}$ & \\
\hline A & 98 & 15,0 & 1,7 & 3,4 & 1,84 & 0,41 & 0,05 & 0,12 & 1,048 \\
\hline B & 149 & 28,5 & 5,4 & 7,0 & 0,15 & 0,08 & 0,04 & 0,07 & 1,023 \\
\hline C & 173 & 16,5 & 3,8 & 4,4 & 0,04 & 0,05 & 0,02 & 0,08 & 1,008 \\
\hline Teores médios & 140 & 20,0 & 3,6 & 4,9 & 0,67 & 0,18 & 0,04 & 0,09 & 1,026 \\
\hline
\end{tabular}

Os resultados representam médias de duplicatas.

TABELA 6. Concentrações de elementos minerais em sucos concentrados de manga

\begin{tabular}{lrrrrrrrrrr}
\hline Marca & \multicolumn{7}{c}{ Teores de elementos minerais $(\mathrm{mg} / 100 \mathrm{~g})$} & & $\begin{array}{r}\text { Densidade } \\
\left(\mathrm{g} / \mathrm{cm}^{3}\right)\end{array}$ \\
\hline & $\mathrm{K}$ & $\mathrm{Na}$ & $\mathrm{Ca}$ & $\mathrm{Mg}$ & $\mathrm{Fe}$ & $\mathrm{Zn}$ & $\mathrm{Cu}$ & $\mathrm{Mn}$ \\
\hline $\mathrm{B}$ & 50 & 25,3 & 4,5 & 10,2 & 0,15 & 0,08 & 0,04 & 0,06 & 1,043 \\
$\mathrm{C}$ & 131 & 9,2 & 6,1 & 10,3 & 0,22 & 0,39 & 0,07 & 0,27 & 1,035 \\
& & & & & & & & & \\
\hline \multicolumn{2}{l}{ Teores médios } & 140 & 17,2 & 5,3 & 10,2 & 0,18 & 0,24 & 0,06 & 0,16 & 1,039 \\
\hline Os resultados representam médias de duplicatas. & & & & & &
\end{tabular}

TABELA 7. Concentrações de elementos minerais em sucos concentrados de maracujá

\begin{tabular}{|c|c|c|c|c|c|c|c|c|c|}
\hline \multirow[t]{2}{*}{ Marca } & \multicolumn{7}{|c|}{ Teores de elementos minerais $(\mathrm{mg} / 100 \mathrm{~g})$} & \multicolumn{2}{|r|}{$\begin{array}{r}\text { Densidade } \\
\left(\mathrm{g} / \mathrm{cm}^{3}\right)\end{array}$} \\
\hline & $\mathrm{K}$ & $\mathrm{Na}$ & $\mathrm{Ca}$ & $\mathrm{Mg}$ & $\mathrm{Fe}$ & $\mathrm{Zn}$ & $\mathrm{Cu}$ & $\mathrm{Mn}$ & \\
\hline A & 272 & 28,5 & 5,6 & 11,2 & 0,94 & 1,7 & 0,06 & 0,11 & 1,037 \\
\hline B & 270 & 28,4 & 4,6 & 12,6 & 0,27 & 1,3 & 0,10 & 0,14 & 1,045 \\
\hline $\mathrm{C}$ & 214 & 19,7 & 3,1 & 10,1 & 0,37 & 0,2 & 0,08 & 0,11 & 1,044 \\
\hline $\mathrm{D}$ & 255 & 2,3 & 3,7 & 10,2 & 0,18 & 0,2 & 0,08 & 0,13 & 1,046 \\
\hline E & 180 & 22,4 & 3,6 & 6,6 & 0,40 & 0,1 & 0,04 & 0,08 & 1,051 \\
\hline Teores médios & 238 & 20,2 & 4,1 & 10,1 & 0,43 & 0,7 & 0,07 & 0,11 & 1,045 \\
\hline
\end{tabular}

TABELA 8. Concentrações de elementos minerais em sucos concentrados de pitanga

\begin{tabular}{|c|c|c|c|c|c|c|c|c|c|}
\hline \multirow[t]{2}{*}{ Marca } & \multicolumn{8}{|c|}{ Teores de elementos minerais $(\mathrm{mg} / 100 \mathrm{~g})$} & \multirow{2}{*}{$\begin{array}{l}\text { Densidade } \\
\qquad\left(\mathrm{g} / \mathrm{cm}^{3}\right)\end{array}$} \\
\hline & $\mathrm{K}$ & $\mathrm{Na}$ & $\mathrm{Ca}$ & $\mathrm{Mg}$ & $\mathrm{Fe}$ & $\mathrm{Zn}$ & $\mathrm{Cu}$ & $\mathrm{Mn}$ & \\
\hline E & 70 & 18,9 & 4,2 & 5,0 & 0,56 & 0,07 & 0,03 & 0,03 & 1,020 \\
\hline
\end{tabular}

Os resultados representam médias de duplicatas.

Os sucos de fruta analisados mostraram-se uma boa fonte de potássio, o que é usual em frutas e hortaliças, variando de 70 a $606 \mathrm{mg} / 100 \mathrm{~mL}$ nos sucos concentrados (Tabelas 2-8). A contribuição dos sucos para a dieta de crianças, após a diluição recomendada pelo fabricante e considerando a ingestão diária de um copo de $300 \mathrm{~mL}$, é elevada quanto a potássio para todos os sucos examinados, atingindo de 170 a $930 \%$ da necessidade diária recomendada (RDA) (Tabela 9) [7]. Para adultos, os teores de potássio nas mesmas condições, variaram de 4 a 13 vezes o RDA (Tabela 10) [7]. 
As concentrações de sódio nos sucos concentrados de frutas, por sua vez, devem-se principalmente à utilização de conservadores sob a forma de sais de sódio (geralmente metabissulfito e benzoato de sódio) e, em segundo lugar ao teor do mineral já naturalmente presente na fruta. A ingestão de sódio pela população é normalmente alta devido ao consumo de sal e alimentos processados. Teores elevados de sódio na dieta não são recomendados para pacientes hipertensivos. Sabe-se que grupamentos humanos cuja dieta é hipossódica apresentam índices de hipertensão mais baixos que populações consumidoras de dietas com níveis altos de sódio [8]. Uma relação alta para a razão potássio/sódio é também favorável do ponto de vista cardiovascular [4]. Os sucos de fruta comerciais examinados apresentaram niveis baixos de sódio, variando entre 9 a $41 \mathrm{mg} / 100 \mathrm{~mL}$ (Tabelas 2-8).

TABELA 9. Contribuição nutricional média estimada para uma criança de um copo de $300 \mathrm{~mL}$ de suco após a diluição recomendada pelo fabricante

\begin{tabular}{lllllllllr}
\hline \multicolumn{10}{c}{ \% ingestão diária recomendada } \\
\hline Suco & Diluição & \multicolumn{1}{c}{$\mathrm{K}$} & $\mathrm{Ca}$ & $\mathrm{Mg}$ & \multicolumn{1}{c}{$\mathrm{Fe}$} & $\mathrm{Zn}$ & $\mathrm{Cu}$ & $\mathrm{Mn}$ \\
\hline Abacaxi & $1: 4$ & 900 & 3 & 9 & 6 & 6 & $3-6$ & 38 \\
Acerola & $1: 4$ & $520-390$ & 1 & 3 & 12 & 6 & $1-2$ & 7 \\
Caju & $1: 10$ & $0-230$ & $<1$ & 2 & 1 & $<1$ & $1-2$ & 2 \\
Goiaba & $1: 3-5$ & $700-930$ & $<1$ & 3 & 7 & 4 & $2-4$ & 8 \\
Manga & $1: 3$ & $700-930$ & 1 & 6 & 2 & 2 & $3-6$ & 14 \\
Maracujá & $1: 9-13$ & $400-530$ & 2 & 2 & 1 & 2 & $1-2$ & 3 \\
Pitanga & $1: 3$ & $350-470$ & $<1$ & 3 & 6 & $<1$ & $2-3$ & 2
\end{tabular}

Foram usadas as recomendações para crianças de 1 a 10 anos, ou quando as recomendações estavam expressas por quilo de peso, os RDAs foram calculados para crianças com $30 \mathrm{~kg}$ de peso.

TABELA 10. Contribuição nutricional média estimada para um adulto de um copo de $300 \mathrm{~mL}$ de suco após a diluição recomendada pelo fabricante

\begin{tabular}{llrrrrrrrrrrr}
\hline & \multicolumn{10}{c}{ \% ingestão diária recomendada } \\
\hline & \multicolumn{1}{c}{$\mathrm{K}$} & $\mathrm{Ca}$ & $\mathrm{Mg}$ & \multicolumn{2}{c}{$\mathrm{Fe}$} & \multicolumn{2}{c}{$\mathrm{Zn}$} & $\mathrm{Cu}$ & $\mathrm{Mn}$ \\
\hline Suco & Diluição & & & & $\mathrm{h}$ & $\mathrm{m}$ & $\mathrm{h}$ & $\mathrm{m}$ & & \\
\hline Abacaxi & $1: 4$ & 5 & 3 & 5 & 10 & 6 & 1 & 6 & $1-3$ & $9-22$ \\
Acerola & $1: 4$ & 2 & 1 & 2 & 12 & 8 & 4 & 5 & 1 & 4 \\
Caju & $1: 10$ & $<$ & $<1$ & 1 & $<1$ & $<1$ & $<1$ & $<1$ & 1 & 1 \\
Goiaba & $1: 3-5$ & 4 & $<1$ & 3 & 7 & 4 & 3 & 3 & $1-2$ & $2-4$ \\
Manga & $1: 3$ & 4 & 1 & 3 & 2 & 1 & 2 & 2 & $1-4$ & $3-8$ \\
Maracuján & $1: 9-13$ & 2 & 2 & 1 & 1 & 1 & 1 & 2 & 2 & $2-8$ \\
Pitanga & $1: 3$ & 2 & $<1$ & 2 & 6 & 4 & $<1$ & $<1$ & $1-2$ & 1 \\
\hline
\end{tabular}

$\mathrm{h}$ - homem adulto, $\mathrm{m}$ - mulher adulta em idade fértil.

Para crianças, os sucos diluídos de abacaxi e acerola podem fornecer 6 a 12\% da RDA (ingestão diária recomendada) de ferro, respectivamente [7]. O manganês aparece em 300mL de sucos já diluídos de abacaxi, manga, goiaba e acerola com 38, 14, 8 e $7 \%$ da RDA, respectivamente [7]. Magnésio mostra uma variação entre um máximo de $9 \%$ da RDA em abacaxi e $2 \%$ em maracujá e caju. As concentrações de zinco e cobre, por sua vez, estão entre menos de $1-2 \%$ do RDA em sucos de caju e pitanga, a $2-6 \%$ nos demais sucos $(\mathrm{Ta}-$ bela 9) [7]. Note-se que os cálculos realizados não levam em conta a biodisponibilidade, a qual é afetada por fatores individuais, presença de inibidores ou compostos com ação sinérgica no alimento, solubilidade, concentração e estado de oxidação do mineral. Em geral, no entanto, minerais são mais absorvidos pelo organismo através de bebidas, pois nestas, há maior solubilidade dos minerais e menor concentração de inibidores. Dependendo do mineral, a taxa de absorção em líquidos varia de 14 a $76 \%$ [2]. No caso de adultos, as quantidades de minerais ofertadas pelos sucos não são despreziveis (Tabela 10), principalmente para magnésio e ferro, na maioria dos sucos.

Mudanças na composição nutricional dos sucos podem ocorrer durante o processamento e principalmente durante a concentração ou outros tipos de exposição ao calor. Sabe-se também que sucos de fruta raramente têm uma composição constante devido à variação natural na composição das frutas. Para investigar estas diferenças, suco de maracujá de dois lotes diferentes, de uma mesma marca, foram analisados (Tabela 11) evidenciando uma variabilidade de 2 a $20 \%$ nos resultados para os diversos elementos analisados. A variação na concentração de minerais que ocorre entre lotes foi também relatada por MORGANO, QUEIROZ \& FERREIRA [6].

TABELA 11. Sucos concentrados de maracujá de lotes diferentes de uma mesma marca

\begin{tabular}{|c|c|c|c|c|c|c|c|c|c|}
\hline \multirow[t]{2}{*}{ Marca } & \multicolumn{8}{|c|}{ Teores de elementos minerais $(\mathrm{mg} / 100 \mathrm{~g})$} & \multirow{2}{*}{$\begin{array}{r}\text { Densidade } \\
\left(\mathrm{g} / \mathrm{cm}^{3}\right)\end{array}$} \\
\hline & $\mathrm{K}$ & $\mathrm{Na}$ & $\mathrm{Ca}$ & $\mathrm{Mg}$ & $\mathrm{Fe}$ & $\mathrm{Zn}$ & $\mathrm{Cu}$ & $\mathrm{Mn}$ & \\
\hline C & 214 & 19,7 & 3,1 & 10,1 & 0,37 & 0,21 & 0,08 & 0,11 & 1,044 \\
\hline C & 209 & 20,8 & 2,3 & 10,9 & 0,28 & 0,23 & 0,07 & 0,09 & 1,041 \\
\hline
\end{tabular}

Os resultados representam médias de duplicatas.

TABELA 12. Resultados de estudos de composição do suco de abacaxi

\begin{tabular}{lcccccccccc}
\hline & \multicolumn{1}{c}{ Teores de elementos minerais } & \multicolumn{2}{l}{$(\mathrm{mg} / 100 \mathrm{~g})$} \\
\hline Autores & $\mathrm{K}$ & $\mathrm{Na}$ & $\mathrm{Ca}$ & $\mathrm{Mg}$ & $\mathrm{Fe}$ & $\mathrm{Zn}$ & $\mathrm{Cu}$ & $\mathrm{Mn}$ & $\mathrm{P}$ \\
\hline & & & & & & & & & \\
Presente trabalho( $\left.{ }^{*}\right)$ & 62 & 4,6 & 7,5 & 5,2 & 0,2 & 0,25 & 0,02 & 1,5 & - \\
{$[5]$} & 121 & 17,2 & 15,6 & 11,3 & 0,3 & 0,06 & 0,03 & 1,1 & 7,6 \\
{$[3]$} & 106 & 31 & 16 & - & 0,4 & - & - & - & 13 \\
{$[5]$} & 53 & 8 & 8 & 6 & 0,2 & 0,1 & 0,02 & 0,7 & 1 \\
{$[1]$} & 91 & 5,7 & 15,2 & 12,9 & 0,2 & 0,07 & 0,05 & 0,9 & 3,1 \\
\hline
\end{tabular}

(*) Teores médios (Tabela 1) divididos pela diluição aconselhada pelo fabricante.

De uma maneira geral, sucos de frutas têm sido pouco estudados. Na Tabela 12 vemos resultados de análises de suco de abacaxi realizadas no Brasil, Espanha e Reino Unido, um dos poucos tipos de suco para o qual dados de composição podem ser encontrados. Uma grande variação pode ser observada entre os resultados. Esta variação pode ser atribuída a diferenças entre cultivares, grau de maturidade e procedência da fruta, assim como diferenças no processamento. Os resultados desta tabela demonstram a necessidade de uma massa de dados de tamanho suficiente que permita a obtenção de médias confiáveis, assim como da 
faixa de variação a ser esperada no produto para elaboração de tabelas de composição de alimentos que reflitam a realidade. Por outro lado, a confiabilidade dos dados analíticos é essencial. As fontes primárias dos dados devem demonstrar o controle de qualidade analítico seguido de maneira a comprovar a validade dos resultados exibidos.

\section{4 - CONCLUSÕES}

O presente trabalho determinou oito elementos minerais ( $\mathrm{K}, \mathrm{Na}, \mathrm{Ca}, \mathrm{Mg}, \mathrm{Fe}, \mathrm{Zn}, \mathrm{Cu}, \mathrm{Mn}$ ) em sucos concentrados comerciais de frutas nacionais provenientes de 7 fabricantes. Todas as marcas analisadas eram de circulação nacional. Todos os sucos analisados mostraram-se uma boa fonte de potássio para adultos e crianças. Paralelamente exibiram níveis baixos de sódio. Para crianças, 300mL diários dos sucos de abacaxi e acerola, diluídos de acordo com as instruções do fabricante, podem fornecer 6 a $12 \%$ da ingestão diária recomendada de ferro, respectivamente. O suco diluído de abacaxi pode ainda contribuir para a dieta infantil com $38 \%$ e 9\% da ingestão diária recomendada de manganês e magnésio, respectivamente. Para adultos, 300mL dos sucos diluídos de abacaxi e acerola podem contribuir com 6 a 10\% e 8 a 12\%da ingestão diária recomendada de ferro, respectivamente. Para outros minerais as contribuições para a dieta são marginais, mas não desprezíveis. O mesmo pode ser dito com relação aos sucos das demais frutas. Os resultados descritos recomendam o consumo de sucos de frutas tanto por crianças como por adultos.

\section{5 - REFERÊNCIAS BIBLIOGRÁFICAS}

[1] CÁmARA, M.; DIEZ, C.; TORIJA, E. Chemical characterization of pineapple juices and nectars. Principal components analysis. Food chemistry, v. 54, p. 93-100, 1995.
[2] EKMEKCIOGLU, C. Intestinal bioavailability of minerals and trace elements from milk and beverage in humans. Nahrung, v. 44, p. 390-397, 2000.

[3] FRANCO, G. Tabela de Composição Guímica dos Alimentos. 9a . ed. Livraria Atheneu, Rio de Janeiro, 1992.

[4] HADDY, F.J.; PANNANI, M.B. Role of dietary salt in hypertension. Journal of the American College of Nutrition, v. 14, p. 428-438, 1995.

[5] HOLLAND, B.; WELCH, A.A.; UNWIN, I.D.; BUSS, D.H.; PAUL, A.A.; SOUTHGATE, D.A.T. The composition of foods. The Royal Society of Chemistry and Ministry of Agriculture, Fisheries and Food, Cambridge and London, 1991.

[6] MORGANO, M.A.; QUEIROZ, S.C.N.; FERREIRA, M.M.C. Determinação dos teores de minerais em sucos de frutas por espectrometria de emissão óptica em plasma indutivamente acoplado. Ciênc. Tecnol. Aliment., v. 19, p. 344-348, 1999.

[7] NATIONAL RESEARCH COUNCIL. Recommended dietary allowances. National Academy Press, Washington, D.C., 1989.

[8] PAGE, L.B. Hypertension and atherosclerosis in primitive and acculturating societies. In: HUNT, J.C. (ed.) Hypertension update. New Jersey: HLS, 1979. v. 1, p. 1-12.

[9] RIZZON, L.A.; MIELE, A. Características analíticas de sucos de uva elaborados no Rio Grande do Sul. Boletim da Sociedade Brasileira de Ciência e Tecnologia de Alimentos, v. 29, p. 129-133, 1995.

[10] SGABIERI, W.C. Alimentação e nutrição: Fator de saúde e desenvolvimento. UNICAMP/Almed, Campinas/São Paulo, 1987.

\section{6 - AGRADECIMENTOS}

A autora K.S. agradece a bolsa concendida pelo Serviço de Apoio ao Estudante da Universidade Estadual de Campinas e as autoras A. M. M.M. e V.A.M. agradecem as bolsas de Iniciação Científica concedidas pelo Conselho Nacional de Pesquisa e Desenvolvimento (CNPq). 